\title{
Civilisations
}

Revue internationale d'anthropologie et de sciences

humaines

52-1 | 2004

Transmission des savoirs et interactions culturelles

\section{L'art celtique de La Tène et les traditions culturelles indo-européennes}

Paul-Louis van Berg

(2) OpenEdition

Journals

Édition électronique

URL : http://journals.openedition.org/civilisations/741

DOI : $10.4000 /$ civilisations.741

ISSN : 2032-0442

Éditeur

Institut de sociologie de l'Université Libre de Bruxelles

Édition imprimée

Date de publication : 1 mai 2004

Pagination : 49-60

ISSN : 0009-8140

Référence électronique

Paul-Louis van Berg, "L'art celtique de La Tène et les traditions culturelles indo-européennes »,

Civilisations [En ligne], 52-1 | 2004, mis en ligne le 28 janvier 2009, consulté le 02 juin 2020. URL

http://journals.openedition.org/civilisations/741 ; DOI : https://doi.org/10.4000/civilisations.741

(c) Tous droits réservés 


\title{
L'art celtique de La Tène et les traditions culturelles indo-européennes
}

\author{
PAUL-LOUIS VAN BERG
}

Les arts des cultures indo-européennes septentrionales se distinguent de ceux de la Méditerranée par leurs formes mêmes. Plus généralement, les productions de ces deux univers reflètent des manières d'être distinctes : organisation territoriale, structures sociales, ordonnance du savoir, conception de la réalité, philosophie de l'image ou ancrage dans le temps. Les choix esthétiques des uns et des autres s'intègrent à leurs visions du monde respectives. Ainsi, les arts de la civilisation de La Tène et de ses épigones dans les îles Britanniques reflètent-ils assez précisément les manières de penser qui transparaissent dans d'autres registres de la culture et en particulier dans le langage poétique.

\section{LA QUESTION}

Lignes droites, décors des poteries et des vases métalliques à grandes figures géométriques, personnages et animaux en ronde bosse auxquels s'ajoutent, dans la province orientale, quelques rares scénographies, montrent que l'art hallstattien d'Europe centrale, réputé appartenir au monde celtique, doit ses modes d'expression à la Méditerranée centrale et aux Balkans. De multiples sources et influences ont été proposées pour expliquer ce phénomène : scènes narratives grecques du VIII ${ }^{\mathrm{e}}$ siècle, décors du Géométrique grec de la première moitié du VII ${ }^{\mathrm{e}}$ siècle, poteries de Bosut (VIII'-VII ${ }^{\mathrm{e}}$ s.), de Bessarabie ou d'Italie du Nord (Megaw, Megaw 1989 : 33). Le réalisme figuratif de l'art adriatique des situles, sans doute d'inspiration étrusque (Megaw, Megaw 1989 : 37), relève du même genre de rapport à l'image.

A la différence de celle de Hallstatt, bon nombre des cultures qui s'expriment en langues indo-européennes font subir de multiples transformations à une imagerie qu'elles empruntent à la Chine, à l'Iran, à la Mésopotamie, à la Grèce ou à Rome. C'est le cas des Scythes, des Grecs du VIII' ${ }^{\mathrm{e}}$ siècle, des Celtes de la civilisation de La Tène et de leurs épigones dans les îles Britanniques ( $V^{\mathrm{e}} \mathrm{s}$. av. n. è.-XII ${ }^{\mathrm{e}} \mathrm{s}$. de n. è.), des Anglo-Saxons ou des Germains, du bas-empire aux Vikings. Toutes ces traditions adaptent à leurs besoins propres un mode de communication visuelle méridional. 
Ainsi par exemple, les différences qui opposent les arts celtiques à ceux de la Méditerranée centrale et orientale sont-elles immédiatement perceptibles. Au sud, le choix d'un « réalisme plus ou moins idéalisé », incompatible avec les arts géométriques, fait que la reconnaissance immédiate des figures pose peu de problèmes et que la représentation suscite avant tout des questions de chronologie, de sémantique et de contexte. Au nord, dans les arts figurés de la civilisation de La Tène et ceux qui en dérivent, distorsion et dislocation de l'image, dissolution du figuratif en formes géométriques, jeux sur la forme et le fond, multiplication des niveaux de lecture et compositions à partir d'un répertoire de figures élémentaires rendent souvent la composition plus difficile à lire.

Dès ses premières manifestations, au $\mathrm{V}^{\mathrm{e}}$ siècle avant notre ère, l'art de La Tène emprunte des figures anthropomorphes, zoomorphes et phytomorphes à l'Italie ou à la Grèce mais, très vite, plutôt que de s'approprier l'imitation de la nature, les transforme d'étrange manière pour un œil éduqué aux arts classiques. L'image « laténienne » simplement réaliste est rare, comme si la mimêsis était de peu d'intérêt. Les scénographies sont absentes jusqu'à la conquête romaine. Quant aux figures individuelles, leurs visages sont impersonnels et, ni les proportions corporelles, ni l'intégrité de l'espèce ne sont toujours respectées. Au contraire, figures humaines et animales sont fréquemment déformées, décomposées, combinées avec une surprenante virtuosité à des formes géométriques curvilignes, interprétées comme végétales, tandis que des compositions purement géométriques au premier abord, laissent transparaître des faces humaines ou animales évanescentes. Dans leur refus de l'objectivité figurative, soumis à des contraintes formelles qui ne sont pas celles du monde classique et orientés vers d'autres significations, les messages de l'art laténien sont anhistoriques. La perception de leur référent nous échappe, laissant traîner un parfum de mystère et d'insatisfaction.

Dans cette « forêt de symboles », du romantisme à nos jours, les historiens de l'art ont senti confusément des correspondances entre les principaux traits de l'art de La Tène et l'esprit des Celtes. Ils ont perçu des affinités entre cet art et les affirmations des quelques notices antiques concernant Gaulois et Galates ou la vision du monde reflétée par les littératures médiévales irlandaise et galloise. Pourtant, les spécialistes ont manifestement du mal à définir l'art de La Tène.

Si l'horloge a tourné depuis le temps où Duval écrivait que l'art des Celtes se caractérise par « l'attrait du fantastique, la familiarité avec le surnaturel, le goût du rêve... » et « la projection instinctive sur le monde sensible d'une vision formée au plus profond de soi »(Duval $1977: 8-10)$, les explications n'en restent pas moins embarrassées et parfois absurdes.

Associant l'ambiguïté de certaines images au goût des Celtes médiévaux pour les récits de métamorphoses, Ruth et Vincent Megaw assignent notre incompréhension à notre seule altérité culturelle :

"This fluidity of concept is that which in other 'wonders' enabled young girls to change into swans, or brave heroes into a salmon, an eagle or a hawk. The same shape-changing ambiguity, of motif rather than myth, is present in the pre-Roman Celtic art where seemingly abstract decoration becomes imbued with human and animal forms reduced to the merest visual formulae. Such ambiguity is only in the mind of the outsider looking in. " (Megaw, Megaw $1989: 22)$. 
Un peu plus tard, Kruta compare l'art laténien à l'Art Nouveau et leur attribue à chacun :

« une préoccupation commune pour l'aspect dynamique des compositions et un goût similaire pour le foisonnement d'éléments divers, empruntés généralement au monde naturel, mais le plus souvent transformés, quelquefois jusqu'à l'abstraction, et imbriqués sans logique évidente. » (Kruta 1991 : 30).

Comment mieux dire que nous ne comprenons pas?

L'année suivante, Eluère voit toujours dans l'art laténien une panoplie de symboles magiques, comme l'avait fait Duval quinze ans plus tôt. En outre, des innovations techniques auraient permis à la courbe de se libérer des arts géométriques répétitifs propres à la civilisation de Hallstatt :

«Au V $V^{e}$ siècle, des innovations techniques permettent aux Celtes de sortir définitivement de l'art géométrique répétitif encore très en vogue au premier âge du fer dans de nombreuses régions. Les lignes se courbent, se libèrent; l'assimilation des influences méditerranéennes est désormais consommée. Des thèmes orientaux sont introduits : l'arbre de vie entouré d'oiseaux, de gardiens monstrueux ou de dragons ; le maître des animaux ; la palmette ; la fleur de lotus ; le thème du masque humain. Présents de la Champagne jusqu'en Bohême et aux confins des Carpates, ils trahissent des modifications profondes dans les croyances et constituent la nouvelle panoplie des symboles magiques qui occuperont inlassablement le répertoire des artistes celtes. » (Eluère 1992 : 57).

En 1994, Raftery se demande s'il ne faut pas accorder aux Celtes plus de responsabilité dans leurs manipulations artistiques, mais en revient finalement à l'idée que le choix des formes est une production de l'inconscient et que l'art nous permet de jeter un furtif coup d'œil au cœur de l'âme celtique :

«It is not always clear, however, if the perceived image was really intended by the artist or if it is no more than the accidental juxtaposition of abstract curves. Or is it at all a deliberate ploy on the part of the craftsman to tantalize and mystify? La Tène art thus supplies us with insights into the Celtic personality which is laden with innuendo, contradictions and double meaning. (...) La Tène art undoub-tedly had a meaning, but that meaning is in most instances lost to us for ever. Through the art, however, we can glance fleetingly into the Celtic soul. » (Raftery 1994 : 163).

La même année, Kruta revoit sa position antérieure :

«...L'art celtique constitue donc un moyen d'approche privilégié de l'univers spirituel des Celtes ... Les traits originaux qui le distinguent de tous les autres arts de l'Antiquité apparaissent dès sa phase initiale. Réticent à toute narration, à toute représentation de scènes, il juxtapose tout au plus des éléments allusifs dont la lecture fournissait probablement à l'initié un support autour duquel il pouvait construire un récit. On retrouve dans cette attitude envers la figuration explicite, définitivement figée et univoque, celle que les druides avaient vis-à-vis de l'écriture, considérée comme impropre à transmettre les textes sacrés. Le rôle de l'image n'était donc pas de reproduire les éléments du 
monde visible, mais d'en illustrer les mécanismes cachés : l'image devient un assemblage de signes symboliques... »(Kruta 1994 : 36).

Enfin, Green a les mêmes soupçons que Raftery quant à la responsabilité des artistes mais, sacrifiant aux modes anglo-saxonnes récentes, ajoute à l'explication une touche de chamanisme, de cannabis et d'animisme :

«Cet art était extrêmement symbolique, peut-être délibérément énigmatique et même cabalistique, ne pouvant être déchiffré que par quelques-uns. S'il s'agit d'un art profondément religieux, nous n'avons aucune chance de parvenir à des certitudes absolues quant à sa signification. La fréquence des motifs empruntés au règne animal pourrait indiquer - comme dans d'autres systèmes animistes - que les animaux étaient considérés comme des créatures capables d'intercéder en faveur des hommes auprès des esprits. (...) L'utilisation qui a été faite du motif, de la forme, de la texture est si complexe qu'on peut se demander si certains artistes n'étaient pas atteints de synesthésie, c'est-à-dire si leurs cinq sens n'étaient pas plus ou moins mélangés au lieu d'être totalement distincts. Les courbes, les spirales et autres motifs, apparemment dessinés au hasard, pourraient, au-delà de leur forme pure, renvoyer à une forme de musique ou de danse, ou encore à des schémas de pensée. Les artistes celtiques utilisaient peut-être des substances hallucinogènes pour aider leur imagination... » (Green 1996 : 117).

On sent bien, à lire les auteurs contemporains, qu'il est difficile de dire pourquoi l'art de La Tène est ce qu'il est. Si la plupart admettent et répètent qu'on ne peut étudier l'art indépendamment de la culture, les essais de mise en contexte ne portent généralement que sur le social et l'économique, mais laissent pendante la question sur le fond. Et ce n'est certainement pas la découverte du sens de l'une ou l'autre production individuelle qui permettra d'y répondre : le sens local ne dévoile que rarement la structure globale. Même si les historiens de l'art et les archéologues apprécient chaque jour davantage le savoir-faire et la cohérence des arts de La Tène et si, dans le courant des années 90 , ils commencent à se débarrasser de la vision romantique de la sensibilité celtique pour se demander s'il ne s'agit pas de quelque chose de plus concerté, à l'exception de Kruta (1994), ils nous renvoient encore régulièrement à la sensibilité, au mystère, à la magie ou aux états altérés de conscience, comme si ce qu'ils voient et décrivent ne pouvait relever d'aucune rationalité.

Tous s'accordent à dire que le sens des compositions est irrémédiablement perdu. Et de fait, sans le support de la parole ou de l'écrit, une quête du sens basée sur des documents aussi peu explicites que ceux de l'art de La Tène a peu de chances d'aboutir. Il semble que, dans le même temps, la question devienne marginale et que les auteurs s'en tiennent à situer ces productions esthétiques dans l'espace, le temps, les milieux économiques et sociaux, à repérer les emprunts à la Méditerranée ou à stigmatiser les évolutions stylistiques. Même Kruta, qui avait pris une position plus précise, n’est pas allé jusqu'au bout de son idée d'art religieux, symbolique et initiatique.

Qu'est-ce qui nous rend donc les arts celtiques si inconcevables? Les peuples «barbares » de l'Europe moyenne n'auraient-ils vraiment produit que des expressions purement intuitives sans trop savoir ce qu'ils faisaient? Nous reprendrons ici la question sur une base paléo-ethnographique plus large pour tenter préciser le cadre 
culturel des transformations auxquelles furent soumises les figures méridionales. En faisant porter l'enquête sur les façons de penser dont le monde celtique a dû hériter, nous pourrons peut-être mieux comprendre la signification socio-culturelle des arts laténiens.

\section{LES INDO-EUROPÉENS : ESPACES ET SOCIÉTÉS}

Lorsqu'elles n'ont pas subi d'acculturation méridionale trop importante, comme celles de Rome, de la Perse ou de l'Inde, les cultures de langue indo-européenne développent, au $1^{\mathrm{er}}$ millénaire avant notre ère, des sociétés morcelées, sans contrôle transversal unifié, et dont les structures spatiales varient d'une région à l'autre. La ville n'existe pas. L'architecture est éphémère et l'allure des constructions change en fonction du substrat ou des préférences régionales : maisons à abside dans les Balkans, rectangulaires en Scandinavie, circulaires dans les îles Britanniques depuis le Néolithique. Les lieux de culte sont généralement installés dans la nature : espaces délimités par quelques pierres, sanctuaires de sommet, grottes ou bois sacrés. La frise et le pavage des espaces ornementaux par de grandes figures géométriques, propres aux vieilles traditions de la Méditerranée, ne s'imposent pas plus que le quadrillage du territoire. A ce découpage variable et relativement flou de l'espace civil et religieux, s'ajoute peu d'intérêt pour un ancrage mesuré dans le temps : les cultures indiennes, scythes, grecques, celtiques ou germaniques ne nous ont pas laissé d'annales historiques, tandis que la renommée transmise par la parole y connaît une grande faveur.

Les dieux n'ont pas élaboré de contrôle détaillé de l'histoire ni fixé pour l'éternité le destin de chacun. La société est partout structurée par des liens personnels de dépendance et la redistribution des richesses se fait par le biais de récompenses personnelles pour services rendus. Il n'y a pas d'économie organisée à grande échelle, même si, au premier millénaire, l'archéologie révèle des échanges à longue distance (Sergent 1995 ; Le Roux, Guyonvarc'h 1991 : 120-126).

Accompagnés et contrôlés par des savants-poètes-voyants, les rois règnent sur des territoires limités qui se ramènent parfois à quelques villages, quelques hameaux, ou au fond d'un golfe. On n'observe pas de recensement ni de levée d'armée systématique. Chaque chef vient au combat avec ses hommes et, le cas échéant, se dispute avec les autres sur la champ de bataille, ce qui empêche toute stratégie globale. On en trouve de beaux exemples dans les épopées indiennes, comme dans l'Iliade ou dans l'irlandaise Razzia des vaches de Cooley.

LE SAVOIR

L'écriture est partout tardive et empruntée aux Akkadiens, aux Minoens, aux Phéniciens, aux Assyriens ou aux Araméens dans le Sud et, secondairement aux Grecs, aux Etrusques et aux Romains dans le Nord. Chez les Grecs mêmes, elle reste longtemps décriée. Chez les Celtes et les Germains, elle ne sert d'abord qu'à des inscriptions funéraires et magiques, ou à d'autres emplois spécialisés. Sauf chez les Hittites et les Mycéniens, directement inspirés par des systèmes palatiaux méditerranéens, on ne trouve en général ni listes, ni inventaires ni comptabilité régulière (Sergent 1995 : 386-387 ; Bottéro et al. 1998 ; Steiner 1994 ; Guyonvarc'h 1997 ; Le Roux, Guyonvarc'h 1986 ; Boyer 1990). 
Les cultures sont orales et l'instruction est le privilège de diverses catégories d'individus qui se partagent inégalement les différentes régions du savoir : poésie, sciences naturelles, droit, magie, médecine, divination, musique et souvent prêtrise (Sergent 1995 : 388). Au terme d'un long apprentissage de nature initiatique misant avant tout sur la mémoire, les détenteurs de ces savoirs sont des poètes-voyants censés communiquer avec les dieux, parler leur langue, chanter sous l'effet de leur inspiration et servir d'intermédiaires entre ceux-ci et les hommes. De multiples témoignages littéraires indiens, grecs, celtiques ou germaniques attestent qu'une partie de leur savoirfaire s'illustre dans leur capacité à monter ou à décoder une poésie savante et obscure, jouant sur la longueur ou le nombre des syllabes, bouleversant l'ordre des mots, farcie d'allitérations, de jeux de mots, de constructions grammaticales ambiguës permettant plusieurs lectures, de métaphores à tiroirs, d'énigmes et d'images codées, utilisées à la transmission de récits traditionnels, de la morale pratique, de questions philosophiques ou d'hymnes religieux (Bader 1989 ; Sergent 1995 : 388-389). L'ambiguïté des oracles delphiques dans la tradition littéraire grecque est typique de cette mentalité (Crahay 1974), autant que le style métaphorique et obscur du Rig Veda (Renou 1939, 1954a-b, 1955, 1978) ou la kenning de la poésie scaldique (Boyer 1990).

Depuis une vingtaine d'années, les travaux de Bader (1989), Boyer (1990), Campanile (1977), Guyonvarc'h (1999), Le Roux et Guyonvarc'h (1986), Renou (1978), Ward (1973), Watkins (2001) et d'autres ont mieux mis en lumière la façon de travailler des prêtres-savants-poètes indo-européens et montré leur niveau de formation intellectuelle, leur capacité à manier la langue et à construire des récits. Ces traditions se sont maintenues fort tard dans les littératures antiques et médiévales et j'ai pu montrer, par l'exemple de l'Hymne homérique à Hermès et celui des Assyriaka de Ctésias, comment poètes et pseudo-historiens savaient adapter leur savoir-faire aux conditions du moment, y compris aux changements socio-religieux (van Berg 2001 ; van Berg, Vander Linden 2002).

A la différence des habitudes intellectuelles en Méditerranée orientale, le classement des objets du monde en catégories disjointes est loin d'être le dernier mot de la réflexion. Au contraire, la spéculation noue les niveaux de la réalité plutôt qu'elle ne les sépare, aidée en cela par la nomination, les ambiguïtés des constructions grammaticales, les procédés d'économie ou d'hypercaractérisation, les expressions à double sens, etc. :

« La poésie sacrée des Védas vise à exprimer les correspondances entre les divers niveaux de la réalité, en particulier entre le rituel et le cosmos. » (Renou 1978 : 47 ; voir aussi Renou 1955).

On peut en dire autant de la Grèce où la Théogonie d'Hésiode, par exemple, noue les actions des dieux et le destin des éléments cosmiques. Comme le souligne explicitement Vernant :

«Ouranos, Gaia, Pontos sont bien des réalités physiques, dans leur aspect concret de ciel, de terre, de mer; mais ils sont en même temps des divinités qui agissent, s'unissent et se reproduisent à la façon des hommes. Jouant sur deux plans, la pensée appréhende le même phénomène, par exemple la séparation de la terre et des eaux, simultanément comme fait naturel dans le monde visible et comme enfantement divin dans un temps primordial. » (Vernant 1975 : 116). 
De même, dans l'Edda de Snorri Sturluson, le soleil et la lune sont-ils à la fois des éléments cosmiques et des êtres anthropomorphes (Edda 11-12).

Si les sciences de la nature sont très inégalement et souvent très mal connues, la divination qui, en Mésopotamie par exemple se pratique dans le cadre d'une combinatoire rigoureuse (Bottéro 1974), est toujours inspirée dans le monde indo-européen (Guyonvarc'h 1997 ; Boyer 1978 : 176-180, 1981 : 90, 140 ; 1986 : 89-97 ; Crahay 1974).

\section{LE CORPS ET SON IMAGE}

La variabilité et le flou observés quant à l'organisation de l'espace se reflètent aussi dans la représentation mentale et plastique du corps. Dans les cultures indoeuropéennes archaïques, les dieux ne sont pas représentés parce que, semble-t-il, on ne désire pas préciser leur forme. Les statues divines sont absentes de la Grèce des siècles obscurs, n'existent guère dans le monde celtique avant la conquête romaine, ne sont pas plus fréquentes chez les Germains, les Iraniens ou les Indiens.

Par ailleurs, l'intégrité du corps des dieux et des hommes n'est pas requise. Les mutilations peuvent même qualifier pour des pouvoirs exceptionnels. Ainsi diverses traditions connaissent-elles des aveugles comme Homère et Tirésias, des borgnes comme Odin ou Horatius Cocles, des manchots comme Mucius Scaevola, Tyr ou Nuada-au-bras-d'argent. L'intégrité du squelette n'est pas plus un critère définitoire du bon mort. De la même perspective, la constitution des monstres ne respecte pas obligatoirement l'ordonnance anatomique des vertébrés. Au Luristan, en Grèce, chez les Germains ou les Iraniens, des têtes surnuméraires peuvent apparaître en des sites inattendus du corps humain ou animal. Les naissances hors nature sont également légions. Naître d'un lotus, de gouttelettes de sang répandues sur la terre, de la tête fendue d'une divinité ou des pieds d'un géant n'a rien de choquant : la vraisemblance anatomique n'est pas de rigueur quand il s'agit de coder par des métaphores ou de donner à découvrir par des énigmes les questions de protogénèse et d'autochtonie, les relations du poète et de la richesse ou encore celles de la lance et du frêne.

Les relations qui unissent les faces visible et invisible de l'être ne sont pas univoques. Toute forme animale peut dissimuler une âme humaine, toute forme humaine peut cacher un dieu, un démon ou un magicien :

« Antinoos, frapper un pauvre vagabond! Insensé, quelle honte!... si c 'était par hasard quelqu'un des dieux du ciel!... Les dieux prennent les traits de lointains étrangers et, sous toutes les formes, s'en vont de ville en ville inspecter les vertus des humains et leurs crimes. " (Homère, Odyssée XVII 483-487).

De l'Inde védique à la Grèce, à la Scandinavie et à l'Irlande, des milliers de récits montrent les dieux, les démons et les magiciens maîtrisant les métamorphoses corporelles, les phénomènes météorologiques et les illusions visuelles. Les apparences sont fallacieuses dans un monde où la baguette du magicien peut faire advenir à peu près n'importe quoi. Dans l'Antiquité classique, muées en genre littéraire, les métamorphoses des dieux et des hommes emplissent des volumes entiers. D'un bout à l'autre du domaine indo-européen, illusions visuelles et métamorphoses assurent que les formes du visible sont instables, évanescentes et, somme toute, peu fiables. Comme on pouvait s'y attendre, la figuration s'en ressent.

L'imagerie graphique ou plastique est toujours empruntée à la Méditerranée ou au 
Proche-Orient. Le rapport de la figuration au sensible varie en fonction du substrat, des interactions avec les voisins et du développement propre de chaque culture. De Persépolis à Rome, la pensée de l'Asie antérieure a largement remodelé les cultures de langue indo-européenne méridionales y compris leur imagerie, tandis que Scythes, Germains et Celtes, par exemple, subirent des influences moins précoces et moins pénétrantes, adaptèrent la représentation avec plus de liberté et lui firent rendre leur approche de la réalité, y compris leur méfiance à l'égard du visible.

\section{L'ART LATÉNIEN ET LE TRAVAIL DES POÈTES}

Dès le $V^{e}$ siècle avant notre ère et pendant toute la durée de l'art laténien, la faille, si nette en Méditerranée, qui sépare les arts figurés des arts géométriques est allègrement franchie. Des formes géométriques sont introduites dans la représentation en trois dimensions : c'est le cas de nombreux torques, bracelets, fibules et clavettes à masques ou statues de pierre, avec leurs sourcils et moustaches en « S-couchée » ou en accolade, voire leurs yeux en spirales. D'autres fois, des éléments réalistes entrent dans une composition géométrique. On voit même des figures humaines et animales réalisées à l'aide de formes géométriques. Géométrie et figuration s'interpénètrent parfois si bien que l'objet ne paraît plus appartenir à l'une de ces catégories en particulier.

Les productions géométrico-figuratives montrent aussi que le métier d'orfèvre dut comporter un apprentissage très sérieux du maniement du compas, ainsi que l'illustrent des séries de phalères de bronze ajouré et de plaques ornementales gravées (Lenerz-de Wilde 1977). Ces compositions et d'autres, dominées par l'usage de la courbe et des symétries de rotation ternaires, jouent sur les oppositions de symétries et d'asymétries. Si la géométrie est presque partout présente, elle ne sert pas à exprimer le découpage et le classement de la réalité en objets distincts, mais plutôt le continu, le passage insensible d'un aspect à l'autre. Dans certains cas, l'opposition de la figure et du fond, traditionnelle dans les cultures modernes, est annulée : forme marquée et non marquée sont toutes deux signifiantes.

On connaît aussi des compositions permettant plusieurs niveaux de lecture. Les exemples en sont nombreux, du visage réversible de Bad Dürkheim (Allemagne, fin du Ve-début du IV $V^{\mathrm{e}}$ siècle av. n. è., Green 1996 : 83) à la Petrie Crown, couvre-chef ornemental en fer, découvert en Irlande, dont le décor montre des têtes humaines aux yeux en tête d'oiseau (Raftery 1994 : 156). Un des cas les plus élaborés apparaît sur la pierre de Turoe (Co. Galway), datée vers le début de notre ère. Il s'agit d'un bloc orné, une sorte d'omphalos, apparemment couvert de figures géométriques. En projection plane, un des secteurs principaux du bas-relief montre clairement une tête de porc ou de sanglier, dont le contour est nettement tracé : on en voit les oreilles, les yeux, le groin et les canines. Cette tête, dont la base se trouve au sommet de la pierre n'est pas directement perceptible au spectateur. Chacune des parties de cette face est constituée d'une grande figure (lunule, éventail, spirale, triangle à côtés curvilignes, cercles) en creux sur le fond desquelles se détachent des spirales ou des éventails en relief. L'ensemble révèle trois niveaux de figuration emboîtés, deux en relief et un en creux. Toutes ces figures internes sont également des figures traditionnelles de l'art laténien tardif (Megaw, Megaw 1989 : 208 ; Raftery 1994 : 162). 
Sur le continent et dans le Sud-Est de l'Angleterre, aux $\mathrm{II}^{\mathrm{e}}$ et $\mathrm{I}^{\mathrm{er}}$ siècles avant notre ère, le monnayage imite d'abord des modèles hellénistiques, mais l'image est très vite décomposée en ses parties et les transformations peuvent aller jusqu'à en faire un ensemble de symboles que nous sommes souvent incapables de reconnaître et dont, $a$ fortiori, nous ne comprenons guère les associations.

En fait, presque toutes les caractéristiques envisagées ci-dessus ont été observées par les auteurs, mais sans qu'ils en expliquent la place dans la culture de La Tène, c'est-à-dire sans qu'ils prennent en compte l'idéologie qui les sous-tend. En effet, l'examen des grands traits des cultures indo-européennes montre que ce qu'on a attribué au goût des Celtes pour la rêverie, la fantaisie, la magie ou la métaphore, s'articule en définitive en une vision du monde cohérente inscrite dans un univers de référence plus large puisqu'il appartient à l'ensemble du monde indo-européen. Dans ce cadre, on peut rapprocher l'imagerie de la culture de La Tène, expression savante d'une approche particulière du monde sensible, du travail des poètes-voyants celtiques et autres, dans ses contenus comme dans ses formes.

L'art laténien cherche le mélange des genres et non leur distinction : la vision de l'artisan noue les différents niveaux de la réalité plutôt qu'elle ne les sépare. Or, les poètes indo-européens produisent ce genre d'effet en racontant une même histoire simultanément au niveau du cosmos, des dieux, du rite et parfois de la vie quotidienne, que ce soit par le jeu des noms propres qui rassemblent éléments cosmiques et anthropomorphes, par l'ambiguïté des relations grammaticales ou par un récit codé. De même, on peut voir dans l'imbrication du figuratif et du géométrique un processus analogue à celui qui soumet la parole courante au carcan de l'expression poétique, à la fois sur le plan rythmique et sur celui de l'ordre des mots. Les mélanges et combinaisons des formes qui en dissolvent le référent offrent de bons équivalents à l'obscurité recherchée de la langue savante.

Les alignements de métaphores décrivant sous des formes diverses le même aspect d'un personnage, tels que nous les voyons sous la plume des auteurs médiévaux irlandais (Guyonvarc'h 1999) sont aussi des moyens de dire la même chose sous des formes différentes, effet que l'art laténien propose régulièrement dans la création de formes identiques au départ de prémisses différentes. Cette attitude s'exprime encore dans les jeux graphiques où la forme et le fond s'engendrent l'un l'autre, soulignant que la même réalité peut apparaître sous de multiples facettes, ainsi que l'illustre dans la littérature irlandaise La navigation de Bran fils de Fébal. Après deux jours de navigation sur l'océan à la recherche du Pays des Femmes, Bran, dans son embarcation, rencontre Manannán sur un char à deux roues tiré par des chevaux. Là où Bran ne voit que la mer, Manannán voit un paysage de prairies et de bosquets, là où l'un voit des saumons tachetés, l'autre voit des veaux et des moutons, et ainsi de suite (Rees, Rees 1961: 314-316, trad. fr. complète dans Guyonvarc'h, 1957)

$\mathrm{Au}$ niveau le plus général, on verra une démarche similaire dans les jeux de décomposition/recomposition de l'image. Une grande liberté à l'égard de l'anatomie accompagne donc des contraintes formelles très lourdes : les canons esthétiques passent par une restructuration du visible traduisant dans l'espace les distorsions que l'expression poétique impose à la langue. Dans le même esprit, Boyer (1990) a souligné l'isomorphisme qui unit les entrelacs de l'art viking à la poésie scaldique. Schiltz (1981), malgré l'absence de textes, avait de même suggéré que la syntaxe de l'art des steppes devait se rapprocher de formes langagières perdues. 
Nous voici donc amenés à la question suivante : la virtuosité des artisans ne nous offre-t-elle pas l'équivalent de la langue des dieux obscure au commun des mortels? Subtil et savant, l'art laténien n'exprime-t-il pas sous forme plastique et graphique une démarche intellectuelle similaire à celle des poètes-voyants de la branche celtique des cultures indo-européennes? Même si d'aucuns pensent que l'institution des druides est tardive en Gaule, nous en savons suffisamment pour assurer le caractère hérité des formes du savoir qu'ils transportent, savoir très élaboré requérant de longues études et s'exprimant entre autres dans la poésie. Seul l'initié, c'est-à-dire celui qui avait reçu l'éducation adéquate était à même d'apprécier pleinement ce type de production. Or, si cette approche de l'expression poétique est un trait hérité, les arts graphiques et plastiques sont liés à des acculturations datables. Nous pouvons donc orienter la flèche du temps et nous assurer que c'est bien l'art qui dépend de la poésie et non l'inverse. Le maniement de la parole aurait ainsi son répondant dans la production esthétique et, en particulier, dans le travail du métal.

Une fois acceptée la parenté de l'art et du langage des savants, on comprend mieux l'affirmation de Kruta selon laquelle les arts de la Méditerranée devaient faire l'objet de la même méfiance et du même refus que l'écriture. En effet, chez les Celtes comme dans d'autres cultures indo-européennes archaïques, le savoir et la parole doivent non seulement demeurer fluides et adaptables aux circonstances, mais aussi rester l'apanage de quelques-uns. Confier la parole à l'écriture ou donner aux arts la transparence, c'eut été détourner l'intérêt du lecteur et du spectateur vers d'autres formes de transmission et de savoir. Les poètes-voyants y auraient probablement laissé une grande partie de leurs privilèges.

\section{CONCLUSION}

Pendant un millénaire, les artisans celtes s'ingénient à détourner les apparences, pour suggérer le mélange, le passage, la transformation, le déformation d'une catégorie en l'autre. Ils ne cherchent pas à traduire dans la matière un monde où les choses sont éternellement identiques à elles-mêmes, mais un monde où tout peut être transformé en tout, ou peu s'en faut. L'intégrité des objets n'est pas nécessaire et n'y a jamais été pensée. S'il y a une maîtrise réelle de l'espace, c'est le pouvoir de le déformer ou de le transformer en créant l'illusion visuelle à la manière des druides et des bardes.

Si l'hypothèse est correcte, l'adaptation des arts de la Méditerranée par la culture de La Tène et ses épigones se comprend comme leur prise en charge par une pensée religieuse institutionnalisée de longue date. Les maîtres à penser du monde celtique auraient donc vu dans les arts méridionaux le moyen de conforter leur position, d'élargir leur gamme d'activités et d'étendre leur influence en munissant les objets de luxe, offerts par les rois, du prestige de la communication poétique.

Paul-Louis van Berg, 


\section{Références bibliographiques}

ANONYME

1994 La razzia des vaches de Cooley, trad. fr. de C.-J. GUYONVARC'H, Paris : Gallimard, « L'aube des peuples $»: 336 \mathrm{p}$.

BADER Fr.

1989 La Langue des Dieux ou l'hermétisme des poètes indo-européen, Pisa : Giardini : 312 p.

BOTTERO J.

1974 Symptômes, signes, écritures en Mésopotamie ancienne, in VERNANT J.-P., VANDERMEERSCH L., GERNET J. et BOTTERO J. et al., Divination et rationalité, Paris : Seuil : 70-197.

BOTTERO J., HERRENSCHMIDT C., VERNANT J.-P.

1998 L'Orient ancien et nous. L'écriture, la raison, les dieux, Paris : Hachette-Littératures « Pluriel» : $230 \mathrm{p}$.

\section{BOYER R.}

1978 Les sagas islandaise, Paris : Payot, « Bibliothèque historique » : $230 \mathrm{p}$.

1981 La religion des anciens Scandinaves, Paris : Payot : 252 p.

1986 Le monde du double. La magie chez les anciens Scandinaves, Paris : L'île verte/Berg international : $222 \mathrm{p}$.

1990 La poésie scaldique, Paris : Editions du « Porte-Glaive » : 252 p.

CAMPANILE E.

1977 Ricerche di cultura poetica indoeuropea, Pisa : Giardini : 144 p.

CRAHAY R.

1974 La bouche de la vérité. Grèce, in VERNANT J.-P., VANDERMEERSCH L., GERNET J., BOTTERO J. et al., Divination et rationalité, Paris : Seuil : 201-219.

DUVAL P.-M.

1977 Les Celtes, Paris : Gallimard, «L’Univers des Formes » : 344 p.

ELUERE C.

1992 L'Europe des Celtes, Paris : Découvertes Gallimard/Réunion des Musées nationaux : 176 p.

GREEN M.J.A..

1996 Le monde celtique, trad. de l'anglais par L. ECHASSERIAUD, Paris : Flammarion : 176 p.

GUYONVARC'H C.-J.

1957 La navigation de Bran, fils de Febal, Ogam IX : 304-309

1997 Magie, médecine et divination chez les Celtes, Paris : Payot et Rivages : 418 p.

1999 Le dialogue des deux sages, Paris : Payot, «Bibliothèque scientifique » : $188 \mathrm{p}$.

KRUTA V.

1991 La redécouverte des anciens Celtes, in MOSCATI S., FREY O.H., KRUTA V., RAFTERY B., SZABO M. (éds), Les Celtes, Venise : Bompiani : 29-34.

1994 Les Celtes : une redécouverte, L'Archéologue 3 (février) : 1-66.

LE ROUX F., GUYONVARC'H C.-J.

1986 Les druides, Rennes : Ouest-France, « De mémoire d'homme : l'histoire » : 448 p.

\section{LENERZ-DE WILDE M.}

1977 Zirkelornamentik in der Kunst der Latènezeit, München : Beck : 142 p., 15 figs., 10 cartes, 68 pls.

MEGAW R., MEGAW V.

1989 Celtic Art from its beginnings to the Book of Kells, London : Thames and Hudson : 288 p. 


\section{RAFTERY B.}

1994 Pagan Celtic Ireland. The Enigma of the Irish Iron Age, London : Thames and Hudson : 240 p.

REES A., REES B.

1961 Celtic Heritage. Ancient Tradition in Ireland and Wales, London : Thames and Hudson : 428 p.

RENOU L.

1939 L'ambiguïté du vocabulaire du Rgveda, Journal Asiatique 231 (avril-juin) : 161-235.

1954a Sur l'économie des moyens linguistiques dans le Rgveda, Bulletin de la Société de Linguistique 50 : 47-55.

1954b Sur les traits linguistiques généraux de la poésie du Veda, Silver Jubilee Volume of the ZinbunKagaku-Kenkyusy, Kyoto : Kyoto University : 309-316.

1955 Etudes védiques et paninéennes, Paris : E. De Boccard : T. I, 154 p.

1978 L'Inde fondamentale. Etudes d'indianisme réunies et présentées par Charles Malamoud, Paris : Hermann, collection Savoir : $232 \mathrm{p}$.

1997 Choix d'études indiennes, réunies par Nalini Balbir et Georges-Jean Pinault, Paris : Réimpression de l'Ecole française d'Extrême-Orient n 9: xvi + 1056 p. (2 tomes).

SCHILTZ V.

1981 L'image de l'animal dans l'art des steppes, in SIEBERT G. (éd.), Méthodologie iconographique. Actes du Colloque de Strasbourg 27-28 avril 1979, Strasbourg : AECR: 49-57, pl. XII-XIII.

\section{SERGENT B.}

1995 Les Indo-Européens : histoire, langues, mythes, Paris : Bibliothèque historique Payot : 540 p.

\section{STEINER D.T.}

1994 The Tyrant's Writ. Myths and images of writing in ancient Greece, Princeton, New Jersey : Princeton University Press : 280 p.

\section{STURLUSON S.}

1991 L'Edda. Récits de mythologie nordique, trad. fr. de F.-X. DILLMANN, Paris : Gallimard, « L'aube des peuples » : $234 \mathrm{p}$.

van BERG P.-L.

2001 Hermes and Agni : a Fire-god in Greece?, in HULD M.E., JONES-BLEY K., DELLA VOLPE A., ROBBINS DEXTER A. (éds), Proceedings of the Twelfth Annual UCLA Indo-European Conference. Los Angeles May 26-28, 2000, Washington D.C. : Journal of Indo-European Studies Monograph Series : 189-204.

van BERG P.-L., VANDER LINDEN M.

2002 Ctesias' Assyriaka : Indo-European and Mesopotamian Royal Ideologies, in HULD M.E., JONESBLEY K., DELLA VOLPE A., ROBBINS DEXTER A. (éds), Proceedings of the Thirteenth Annual UCLA Indo-European Conference. Los Angeles November 9-10, 2001, Washington D.C. : Journal of Indo-European Studies Monograph Series : 45-58.

VERNANT J.-P.

1975 Les origines de la pensée grecque, Paris : Presses Universitaires de France : 136 p.

WARD D.

1973 On the Poets and Poetry of the Indo-Europeans, Journal of Indo-European Studies I/4: 127-144.

WATKINS C.

2001 How to kill a dragon : aspects of Indo-European poetics, Oxford-New York : Oxford University Press xiii $+613 \mathrm{p}$. 